\title{
Functional plasticity abnormalities over the lifespan of first-episode patients with major depressive disorder: a resting state fMRI study
}

\author{
Li Yang ${ }^{1}$, An-Hai Wei ${ }^{1,2}$, Tan-Te Ouyang ${ }^{3}$, Zhen-Zhen Cao ${ }^{1}$, Ao-Wen Duan ${ }^{1}$, He-Hua Zhang ${ }^{1}$ \\ ${ }^{1}$ Department of Medical Engineering, Daping Hospital, Army Medical University, Chongqing, China; ${ }^{2}$ College of Communication Engineering \\ of Chongqing University, Chongqing, China; ${ }^{3}$ Department of Biomedical Engineering and Medical Imaging, Army Military Medical University, \\ Chongqing, China \\ Contributions: (I) Conception and design: L Yang; (II) Administrative support: AH Wei, ZZ Cao; (III) Provision of study materials or patients: L Yang, \\ AW Duan; (IV) Collection and assembly of data: TT Ouyang; (V) Data analysis and interpretation: L Yang; (VI) Manuscript writing: All authors; (VII) \\ Final approval of manuscript: All authors. \\ Correspondence to: He-Hua Zhang. Department of Medical Engineering, Daping Hospital, Army Medical University, Chongqing, China. \\ Email: zhanghehua@vip.163.com.
}

Background: Neurodevelopmental and neurodegenerative theories of depression suggest that patients with major depressive disorder (MDD) may follow abnormal developmental, maturational, and aging processes. However, a lack of lifespan studies has precluded verification of these theories. Herein, we analyzed functional magnetic resonance imaging (fMRI) data to comprehensively characterize age-related functional trajectories, as measured by the fractional amplitude of low frequency fluctuations (fALFF), over the course of MDD.

Methods: In total, 235 MDD patients with age-differentiated onsets and 235 age- and sex-matched healthy controls (HC) were included in this study. We determined the pattern of age-related fALFF changes by cross-sectionally establishing the general linear model (GLM) between fALFF and age over a lifespan. Furthermore, the subjects were divided into four age groups to assess age-related neural changes in detail. Inter-group fALFF comparison (MDD vs. HC) was conducted in each age group and Granger causal analysis (GCA) was applied to investigate effective connectivity between regions.

Results: Compared with the HC, no significant quadratic or linear age effects were found in MDD over the entire lifespan, suggesting that depression affects the normal developmental, maturational, and degenerative process. Inter-group differences in fALFF values varied significantly at different ages of onset. This implies that MDD may impact brain functions in a highly dynamic way, with different patterns of alterations at different stages of life. Moreover, the GCA analysis results indicated that MDD followed a distinct pattern of effective connectivity relative to $\mathrm{HC}$, and this may be the neural basis of MDD with agedifferentiated onsets.

Conclusions: Our findings provide evidence that normal developmental, maturational, and ageing processes were affected by MDD. Most strikingly, functional plasticity changes in MDD with different ages of onset involved dynamic interactions between neuropathological processes in a tract-specific manner.

Keywords: Major depressive disorder (MDD); resting-state fMRI; fractional amplitude of low-frequency fluctuation; lifespan

Submitted Dec 23, 2020. Accepted for publication Feb 10, 2021.

doi: $10.21037 / \mathrm{atm}-21-367$

View this article at: http://dx.doi.org/10.21037/atm-21-367 


\section{Introduction}

The brain function abnormalities in major depressive disorder (MDD) have been extensively reported, especially since the emergence of neuroimaging technology (1). Early works have reported deficits localized mostly to the prefrontal cortex, hippocampus, and amygdala mesolimbic dopamine system, which were the result of multiple risk factors such as cognitive processes, stressors, certain socio-demographic factors, and therefore contributed to the neuroplasticity hypothesis of depression $(2,3)$. Recently, abnormal brain connectivity among large-scale functional brain networks has been of particular interest to researchers investigating the pathophysiology of MDD. These studies have revealed hypoconnectivity within the frontoparietal and default networks, as well as disrupted connectivity patterns between the frontal and parietal regions in the dorsal attention network, which leads to deficits in attention, working memory, executive control, facial emotion recognition, and reward processing $(4,5)$. Despite a large number of neuroimaging studies demonstrating the abnormal functional plasticity in depression, a full explanation for depression pathology and lifespan trajectory of brain functions for MDD patients has yet to be established.

It is widely accepted that the structure and function of the brain changes dynamically with age in abnormal individuals (6). Topological analysis of the brain showed inverted-U shaped curves between local efficiency, rich club, and age for healthy people. However, the lifespan trajectory of the brain for depressed patients may differ from normal development (7). The multiple risk factors of depression were thought to be correlated with environmental and genetic factors, whose effects varied throughout the patient's lifespan $(2,3)$. These genetic and environmental factors affect well-being and depressive symptoms greatly in childhood. For adolescents and adults, the substantial contributions from the genetic effects were found to be related to environmental effects (8). Hence, mapping brain changes over the lifespan of the patient may provide insight into the timing and biological nature of the factors underlying the cognitive deficits observed in depression. Previous studies have reported abnormal activation of the left caudate nucleus and the right anterior cingulate gyrus in elderly people (9), and the medial orbitofrontal cortex and superior frontal gyrus in young people (10). However, these studies tended to have small samples of patients at one stage of illness, as well as limited age-windows, which likely obscured interpretations regarding the role of development and aging in functional plasticity disruptions. Also, depressed patients in different age groups were recruited into a unified analysis, which may have obscured dynamic alterations and pathogenesis at different stages of life (11). Given that age strongly influenced the pathogenesis of depression, it is especially interesting and important to describe the lifespan trajectory of brain functions in depressed patients.

Functional magnetic resonance imaging (fMRI) could reflect functional brain alterations, and has been widely used in uncovering the etiology and pathogenesis of MDD (12). Several of the resting-state fMRI (R-fMRI) indices have produced consistent and reliable results corresponding to the functional activities of the brain. The amplitude of low frequency fluctuations (ALFF) and fractional ALFF (fALFF) were used as promising indices to discover biomarkers in basal and clinical research. The averaged square root of the power spectrum between 0.01 and $0.08 \mathrm{~Hz}$ was taken as the ALFF, which examines the amplitude of the regional activity and reflects the intensity of spontaneous neural activity $(13,14)$. However, the ALFF is sensitive to physiological noise. As an improvement to ALFF, fALFF represents the ratio of the power spectrum of low-frequency $(0.01-0.08 \mathrm{~Hz})$ to that of the entire frequency range. Compared with ALFF, it can effectively inhibit the non-specific signals and significantly improve the specificity and sensitivity of detecting spontaneous brain activity (15). Indeed, lifespan studies have indicated obvious differences in evaluating age-related changes between ALFF and fALFF (16). No previous studies have employed fALFF to measure the agerelated changes in depression.

In the present study, we characterized functional plasticity abnormalities over the course of depression using the fALFF indices in a large cohort of MDD patients. We aimed to identify and characterize global and tract-specific age-related trajectories of functional plasticity abnormalities (as measured by fALFF) over the depression lifespan. Based on previously proposed studies, we sought to determine whether the functional plasticity pathology in depression follows a developmental, maturational, and degenerative pattern, and if this pattern varies between depressed individuals and healthy controls (HC). The abnormal age-related trajectories of functional plasticity in MDD prompted us to analyze adolescents, adults, and elderly patients separately at the neural circuit level. Subsequently, the Granger causal analysis (GCA) was conducted to examine the effective connectivity between the neural circuit regions to reveal the brain mechanisms for MDD patients at different periods of disease onset (adolescent- $v$. 
Table 1 General information of the groups

\begin{tabular}{|c|c|c|c|c|}
\hline Information & Adolescent $(\leq 21)$ & Young [22-39] & Middle-aged [40-59] & Old $(\geq 60)$ \\
\hline Number & 39 & 116 & 69 & 11 \\
\hline Female proportion & $77 \%$ & $74 \%$ & $71 \%$ & $73 \%$ \\
\hline Episodicity status & First-episode & First-episode & First-episode & First-episode \\
\hline HAMD & 23.28 & 22.26 & 23.88 & 22.36 \\
\hline \multicolumn{5}{|l|}{$\mathrm{HC}$} \\
\hline Number & 39 & 115 & 70 & 11 \\
\hline Female proportion & $72 \%$ & $74 \%$ & $71 \%$ & $73 \%$ \\
\hline
\end{tabular}

MDD, major depressive disorder; HC, healthy controls; HAMD, Hamilton Depression Scale.

adult- vs. elderly-onset).

We present the following article in accordance with the MDAR reporting checklist (available at http://dx.doi. org/10.21037/atm-21-367).

\section{Methods}

\section{Participants and imaging acquisition}

The initial fMRI data came from the REST-meta-MDD Consortium Data Sharing (http://rfmri.org/REST-metaMDD), which contains R-fMRI data from 1,300 patients with MDD and 1,128 healthy controls (17). The RESTmeta-MDD consortium was established by 25 research groups from 17 Chinese hospitals. The collection of these R-fMRI data was approved by the local institutional review boards. In addition, the age, sex, episodicity (first or recurrent) status, medication status, illness duration, Hamilton depression scale (HAMD) score, and education status were openly shared. Written informed consent was obtained from all participants at their local institution. The analysis in this study was approved by the REST-metaMDD consortium (approval ID: U0040). The study was conducted in accordance with the Declaration of Helsinki (as revised in 2013).

Patients were excluded from this study based on the following criteria: (I) their information (including age, sex, episodicity status, medication status, HAMD score, and education status) was unavailable; (II) patients with HAMD scores below 17 (18); (III) recurrent MDD patients; (IV) patients with poor quality diagnostic images; (V) time points with frame wise displacement $>0.2 \mathrm{~mm}$ (17). Finally, 235 MDD patients were selected. Age- and sex-matched controls without any mental or neurological diseases were gathered from the database. HC exhibiting poor image visual inspection and excessive head movement were also excluded. In total, 235 healthy controls were also included.

The age cut-off for adolescent versus adult analyses was set at 22 years for studying the cortical abnormalities in MDD patients (19). The age-related functional changes from age 40 to 60 tended to be stable, and decreased with aging (20). Considering that the brain varies with age, conflicting results were found in MDD studies. In this study, the 235 MDD patients (range, 13-71 years, and median 35.1 years) were divided into four groups $(\leq 21,22-39$, $40-59, \geq 60$ years). Due to a lack of drug-naive elderly patients, six patients with medicine-treatment and illness duration $\leq 24$ months were included in this study.

The HC (range, 14-72 years, and median 35 years) were divided into four groups according to similar rules. The details of the groups are shown in Table 1. In addition to the number of samples and the proportion of females, the HAMD scores and medication status have also been provided.

\section{fMRI data preprocessing}

Resting state images were preprocessed using Data Processing Assistant for Resting - State fMRI (DPARSF, http://rfmri.org/content/dparsf). Initially, the first 10 functional images of every subject were discarded due to the main-flux saturation effect. Secondly, slice-timing 
correction was performed to correct time differences among the slices during acquisition. Thirdly, the functional images were realigned to the first volume to correct the headmotion with rigid-body transformation. Fourthly, functional images of each subject were co-registered to the T1 structural images and spatially normalized to the Montreal Neurological Institute (MNI) standard template (21). Lastly, spatial smoothing was conducted using a Gaussian kernel with a 6-mm full-width and half-maximum (FWHM) to ensure a high signal-to-noise ratio (SNR).

\section{fALFF calculation}

The calculation formula of fALFF is shown in Eq. [1]. The time series of each voxel were first converted to frequency domain to compute its power spectrum (15). The square root of each frequency in the power spectrum was then obtained. Finally, the sum of the amplitudes for each frequency in the low frequency range $(0.01-0.1 \mathrm{~Hz})$ was divided by the sum of the amplitudes for each frequency in the entire frequency range $(0-0.25 \mathrm{~Hz})$.

$$
\mathrm{fALFF}=\frac{\sum_{0.01 \mathrm{~Hz}}^{0.1 \mathrm{~Hz}} \sqrt{\text { power spectrum }}}{\sum_{0 \mathrm{~Hz}}^{0.25 \mathrm{~Hz}} \sqrt{\text { power spectrum }}}
$$

These fALFF maps were converted into Z-score maps by subtracting the mean and dividing the standard deviation within a whole brain mask for the participant (22).

\section{Statistical analysis}

\section{One-sample $t$-test}

For MDD patients, intra-group analysis was conducted in the four age groups (adolescent, young adults, middleaged adults, and elderly adults) using the one-sample $t$-test $[\mathrm{P}<0.05$, false discovery rate (FDR) corrected, cluster size $\geq 20$ ] (23). Subsequently, results of the one-sample $t$-test in the four groups were overlapped to generate the significant brain changes over a lifespan. The same analysis was conducted for HC. The peak coordinates of significant brain areas were selected as the center of the region of interest (ROI). Subsequently, the fALFF values of the ROI (6 $\mathrm{mm}$ in radius) were extracted to build the general linear model (GLM) with age (24).

\section{GLM}

To determine the influence of age on fALFF abnormalities in MDD patients, we established multiple linear regression equations with gender as the covariate and age as the predictive factor. Concretely, the GLM model could be expressed with the following equations:

$$
\mathrm{fALFF}=\beta_{0}+\beta_{1} \times \text { age }+\beta_{2} \times \text { gender }
$$

$$
\text { fALFF }=\beta_{0}+\beta_{1} \times \text { age }+\beta_{2} \times \text { age }^{2}+\beta_{3} \times \text { gender }
$$

where $A A L F F$ is the dependent variable; age is the independent variable; $\beta_{0}, \beta_{1}, \beta_{2}$, and $\beta_{3}$ are unknown parameters; and gender represents the confound.

\section{Behavioural scale correlation analysis}

In order to investigate the age-related relationship between fALFF and cognition function, we examined correlation between the fALFF values and behavioural parameters, including HAMD scores throughout the lifespan. Pearson's correlation between the fALFF values across the range and HAMD scores was computed $(\mathrm{P}<0.05$, FDR-corrected).

\section{Two-sample $t$-test}

Inter-group analysis (MDD vs. HC) was performed in four age groups using the two-sample $t$-test. The results of the two-sample $t$-test in the four groups were overlapped to obtain the significant brain differences between MDD and HC over the lifespan.

\section{Granger causal analysis (GCA)}

Following the two-sample $t$-test analysis, regions with significantly different fALFF were selected as the ROIs in the four age groups, and the causal interactions between these ROIs were calculated using GCA. GCA is one of the analysis models for effective connectivity, and it emphasizes causality between brain areas (25). The time-series-relevant GCA was conducted using REST-GCA in the REST toolbox (http://www.restfmri.net/forum/REST_V1.8). Intra-group analysis of Granger causality (GC) values between ROIs was performed using the one-sample $t$-test $(\mathrm{P}<0.05$, FDR-corrected).

\section{Results}

\section{One sample t-test results}

For MDD patients, one-sample $t$-test analysis was conducted in the four age groups $(\mathrm{P}<0.05$, FDR-corrected, cluster size $\geq 20$ ). The overlapped image revealed significant fALFF differences in the left medial superior frontal gyrus (MSFG), 

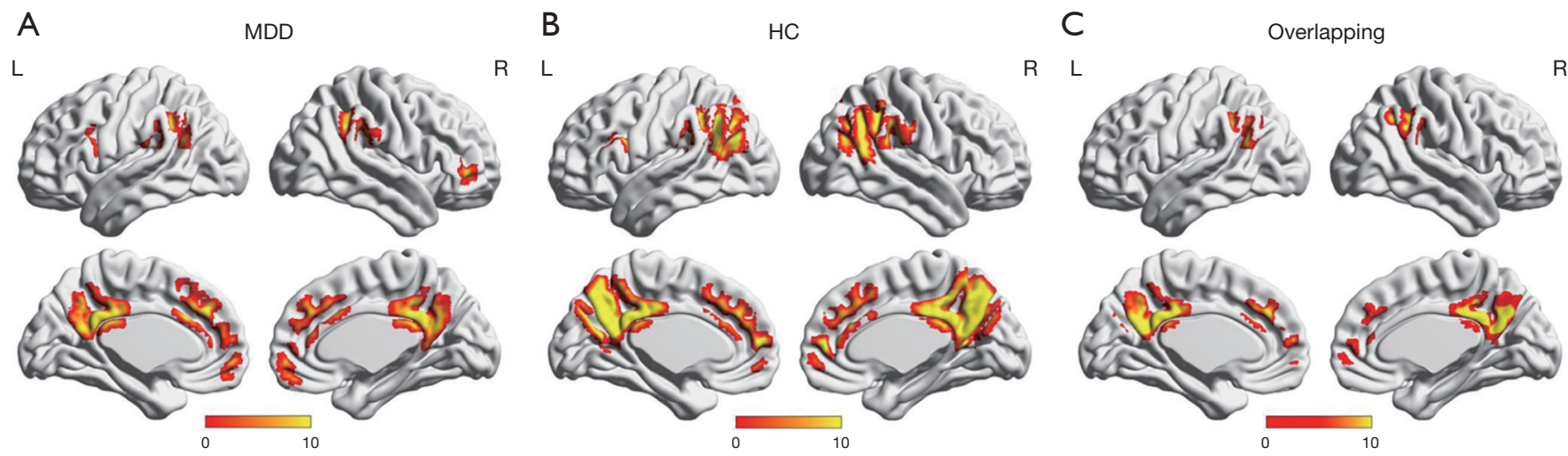

Figure 1 The results of one-sample $t$-test for MDD and HC. (A) The intra-group effect for MDD; (B) whole-brain composite maps for HC; (C) the spatially overlapping regions between MDD and HC. MDD, major depressive disorder; HC, healthy controls.

right precuneus, posterior cingulate cortex (PCC), middle cingulate cortex (MCC), left anterior cingulate cortex (ACC), and supramarginal gyrus (SMG) (see Figure $1 \mathrm{~A}$ and Table 2). For the HC, one-sample $t$-test analysis was performed in the four age groups $(\mathrm{P}<0.05, \mathrm{FDR}$-corrected, cluster size $\geq 20$ ).

Age-related changes in the fALFF were observed in the right MSFG, precuneus, left cuneus, left PCC, left MCC, angular gyrus (AG), right SMG, and left inferior parietal gyrus (IPG) according to the intersection image of the one $t$-test analysis (see Figure $1 B$ and Table 2). The regions detected in both MDD and HC groups were the left ACC, left MSFG, precuneus, left PCC, MCC, left AG, and right SMA (see Figure $1 C$ and Table 2).

\section{Age-related causal interaction analysis results}

The fALFF values extracted from the regions (nine ROIs) that were observed in both MDD and HC groups were modeled over a 60-year period from 13 to 72 years using a GLM with linear and quadratic age terms as the predictive factor (Figure 2). However, there were no significant linear or quadratic age effects for the MDD group. Moreover, only the fALFF within the left MCC was decreased linearly with gender for MDD (Table 3).

Age-squared (the highest order term) was found to be the most significant term in the quadratic model for the HC groups (Figure 2). The fALFF values in the right precuneus, left PCC, MCC, left AG, and right SMG had a significant quadratic age term and demonstrated an age-related $\mathrm{U}$ trend. Specifically, both a significant age and gender effect were discovered within the right SMG (Table 3).

\section{Behavioral scale correlation analysis}

For the entire lifespan, there was no statistically significant correlation between fALFF values and HAMD scores. However, there were significant correlations between the fALFF values and HAMD scores in MDD patients over 50 years of age (Figure 3), which was similar to the results of the HC group (25). The blue line represents the fitted curve between FALFF and HAMD in MDD patients under the age of 50, while the red line represents the fitted curve between AALFF and HAMD over 50 years old. The fALFF in the left MSFG, left AG, and right SMG was negatively correlated with HAMD scores of MDD patients over 50 years old.

\section{Two sample t-test results}

The results of the two-sample tests (MDD vs. HC) are shown in Figure 4 and Table 4 (adolescent, young adults, middle-aged adults, old adults) $(\mathrm{P}<0.05$, FDR-corrected, cluster size $\geq 20$ ). In the adolescent group, relative to $\mathrm{HC}$, the $\mathrm{fALFF}$ in the MSFG were significantly stronger, and the fALFF in the right pallidum were markedly weaker in MDD patients (Figure 4A). In the younger cohort, HC showed notably weaker fALFF within the cerebellum, left middle temporal gyrus (MTG), left parahippocampal gyrus (PHG), and right hippocampus compared to the MDD group, and significantly stronger fALFF in the right middle frontal gyrus (MFG), right SMG, and right IPG (Figure 4B).

For middle-aged people, substantially increased fALFF was observed in the MFG, superior frontal gyrus (SFG), and left precentral gyrus, and decreased fALFF was seen 
Table 2 Distribution of brain regions associated with the one-sample $t$-test results for the MDD and HC groups and the spatially overlapping area between the two groups

\begin{tabular}{|c|c|c|c|c|c|}
\hline Anatomical & Hemisphere & BA & Cluster voxels & MNI $(x, y, z)$ & $t$ value \\
\hline Medial superior frontal gyrus & L & 32 & 97 & $-3,36,36$ & 20.76 \\
\hline Precuneus & $\mathrm{R}$ & 0 & 97 & $3,-48,39$ & 20.26 \\
\hline Posterior cingulate cortex & $\mathrm{L}$ & 23 & 71 & $-3,-45,30$ & 12.67 \\
\hline Middle cingulate cortex & L & 23 & 71 & $-3,-36,36$ & 9.24 \\
\hline Middle cingulate cortex & $\mathrm{R}$ & $23 / 24$ & $47 / 31$ & $3-4236 / 32637$ & $16.14 / 8.21$ \\
\hline Anterior cingulate cortex & $\mathrm{L}$ & 24 & 66 & $0,37,21$ & 8.64 \\
\hline Supramarginal gyrus & L & 39 & 42 & $-51,-51,30$ & 13.84 \\
\hline Medial superior frontal gyrus & $\mathrm{R}$ & 10 & 46 & $3,54,9$ & 17.08 \\
\hline Precuneus & $\mathrm{L}$ & 23 & 187 & $-3,-54,21$ & 44.94 \\
\hline Precuneus & $\mathrm{R}$ & 0 & 175 & $3,-63,36$ & 18.08 \\
\hline Cuneus & L & 0 & 55 & $-3,-72,30$ & 19.79 \\
\hline Posterior cingulate cortex & $\mathrm{L}$ & 23 & 40 & $-3,-50,27$ & 26.71 \\
\hline Middle cingulate cortex & $\mathrm{L}$ & 23 & 35 & $-3,-37,36$ & 16.55 \\
\hline Angular gyrus & L & 7 & 87 & $-39,-63,39$ & 32.51 \\
\hline Angular gyrus & $\mathrm{R}$ & 39 & 66 & $48,-63,30$ & 24.27 \\
\hline Precuneus & $\mathrm{L}$ & 23 & 160 & $-3,-54,24$ & 54.80 \\
\hline Precuneus & $\mathrm{R}$ & 0 & 111 & $3,-60,30$ & 14.15 \\
\hline Posterior cingulate cortex & L & 23 & 60 & $-3,-50,27$ & 42.59 \\
\hline Middle cingulate cortex & L & 23 & 57 & $-3,-39,36$ & 13.84 \\
\hline Middle cingulate cortex & $\mathrm{R}$ & & 38 & $3,-41,36$ & 16.55 \\
\hline Angular gyrus & L & 22 & 83 & $-54,-54,24$ & 16.91 \\
\hline Supramarginal gyrus & $\mathrm{R}$ & 40 & 46 & $57,-48,30$ & 19.92 \\
\hline
\end{tabular}

BA, Brodmann area; MDD, major depressive disorder; HC, healthy controls.

in the calcarine and cuneus, relative to HC (Figure 4C). For elderly people, MDD demonstrated higher fALFF in few voxels of the left inferior temporal gyrus (ITG), and lower fALFF in the left MCC (Figure 4D, $\mathrm{P}<0.001$, cluster size $\geq 5$ ). However, no significant overlapping regions were discovered between the results of the two-sample $t$-tests in 

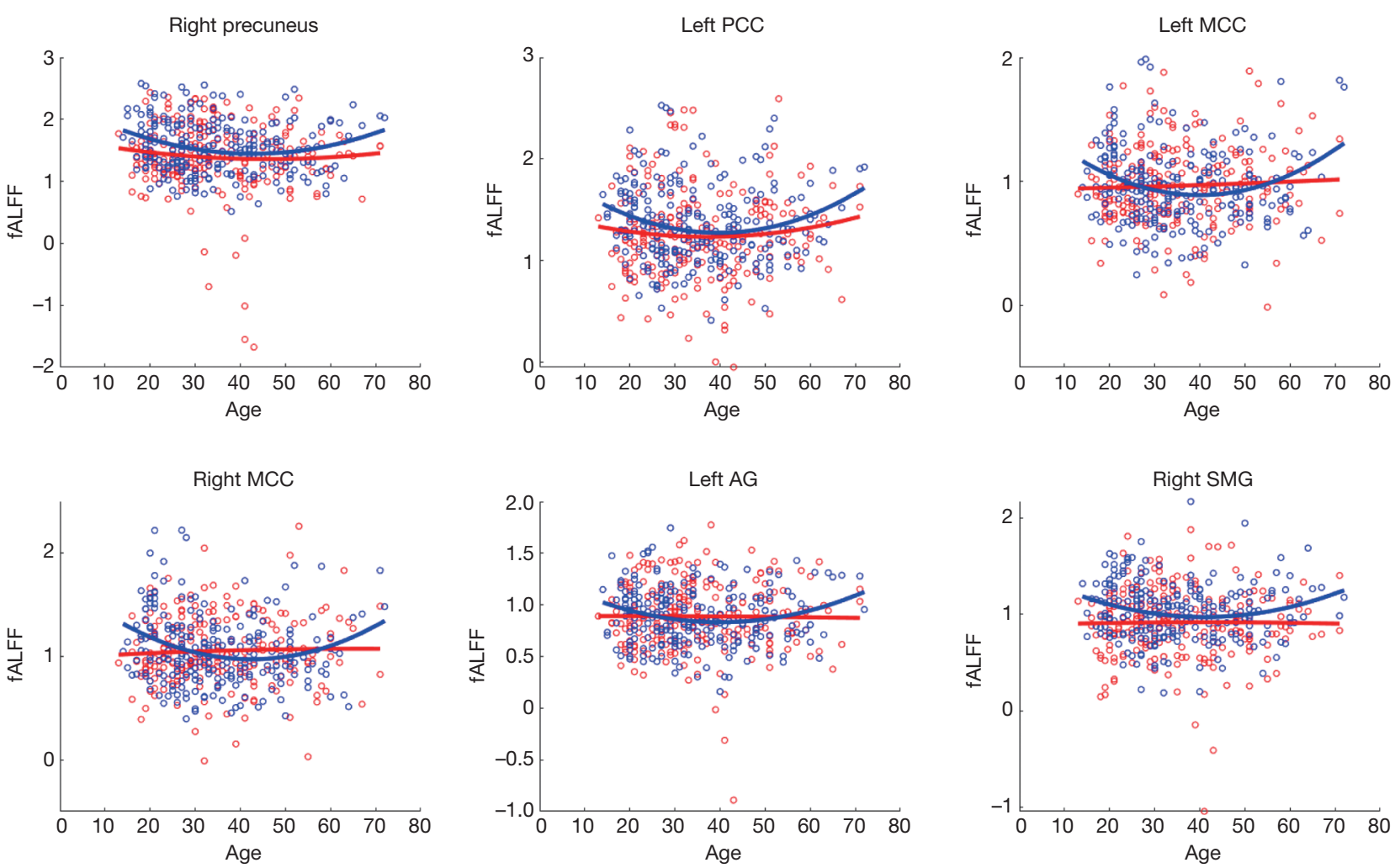

Figure 2 The trends between fALFF and age within the right precuneus, left PCC, MCC, left AG, and right SMG for the MDD and HC groups. The red points and line represent the fALFF value and the fitting curve, respectively, for MDD; the blue points and line represent the fALFF values and the fitting curve, respectively, for HC. HC exhibited significant quadratic age effects. fALFF, fractional amplitude of low frequency fluctuations; MCC, middle cingulate cortex; PCC, posterior cingulate cortex; AG, angular gyrus; SMG, supramarginal gyrus; MDD, major depressive disorder; HC, healthy controls.

Table 3 Statistical parameters of age-related GLM

\begin{tabular}{|c|c|c|c|c|}
\hline fALFF & T-statistic & $P$ & $R^{2} / R$ & Fitting curve \\
\hline Left MCC & 5.06 & 0.0254 & 0.0212 & $y=-0.10331$ gender +1.1452 \\
\hline \multicolumn{5}{|l|}{$\mathrm{HC}$} \\
\hline Right precuneus & 6.00 & 0.0029 & 0.0492 & $y=0.00045 a g e^{2}-0.0389$ age +2.2795 \\
\hline Left MCC & 7.31 & 0.0008 & 0.0592 & $y=0.00041 a g e^{2}-0.0326$ age +1.5395 \\
\hline Right MCC & 7.63 & 0.0006 & 0.0617 & $y=0.00042 a g e^{2}-0.0361$ age +1.7386 \\
\hline Left AG & 4.41 & 0.0131 & 0.0367 & $y=0.00029 a g e^{2}-0.0227$ age +1.2888 \\
\hline Right SMG & 5.63 & 0.0010 & 0.0682 & $\mathrm{y}=0.00030 \mathrm{age}^{2}-0.0246$ age +0.1287 gender +1.2514 \\
\hline
\end{tabular}

Note: $R^{2}$ in $R^{2} / R$ represents the curve fitting degree of the age-related quadratic fitting curve, expressed in italic values; $R$ represents the correlation between fALFF and gender. GLM, general linear model; MDD, major depressive disorder; HC, healthy controls; MCC, middle cingulate cortex; PCC, posterior cingulate cortex; AG, angular gyrus; SMG, supramarginal gyrus. 

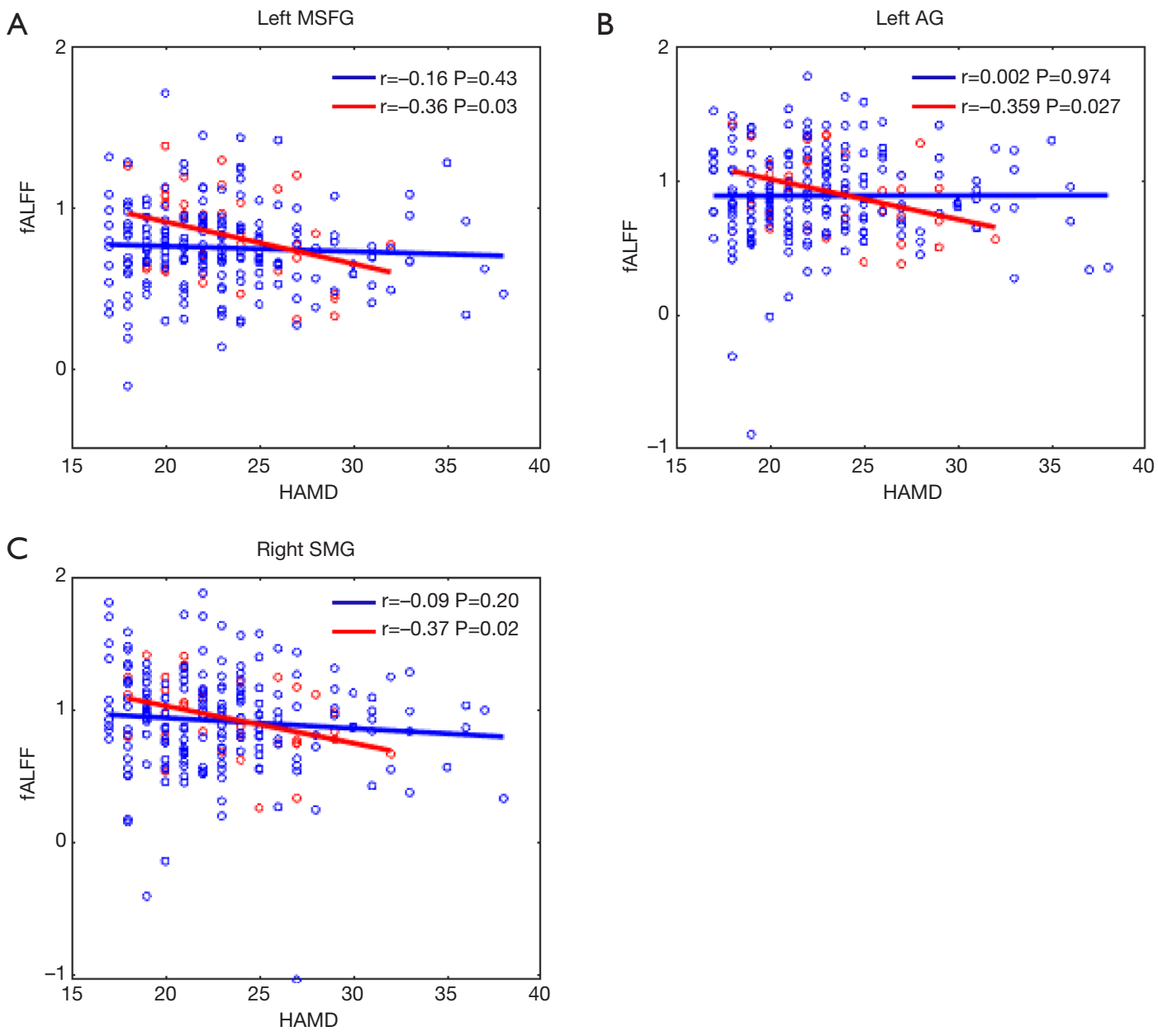

Figure 3 The correlations between fALFF and HAMD scores. (A) Scatter plots demonstrate the relationship between the fALFF within the left MSFG and HAMD scores. (B) Scatter plots demonstrate the relationship between the fALFF within the left AG and HAMD scores. (C) Scatter plots demonstrate the correlation between the fALFF within the right SMG and HAMD scores. The blue line and points indicate the development and maturational process ( $<50$ years), and red line and points indicates the maturational and aging process ( $\geq 50$ years), respectively. fALFF, fractional amplitude of low frequency fluctuations; MSFG, medial superior frontal gyrus; AG, angular gyrus; SMG, supramarginal gyrus.

the four age groups $(\mathrm{P}<0.05$, FDR-corrected, cluster size $\geq 20$ ). In other words, inter-group differences (MDD vs. $\mathrm{HC}$ ) in fALFF values were distinct from each age group.

\section{Causal interactions between regions}

For adolescents, MDD was associated with the MSFG and right pallidum. The GCA indicated the information flows of the right $M S F G \leftrightarrow$ right pallidum were negative in MDD, which was contrary to the HC group (Figure $5 A$ ). For young people, MDD was linked to the cerebellum, left PHG, right hippocampus, right $\mathrm{MFG}$, and right SMG (Figure 5B).
Bidirectional connectivity was observed in MDD individuals between the right hippocampus, right $M F G$, and right SMG (Figure 5B). Specifically, the cerebellum exhibited top-down and bottom-up connectivity to the left PHG, right $\mathrm{MFG}$, and right $\mathrm{SMG}$ in the $\mathrm{HC}$ group.

For middle-aged people, MDD was associated with negative GC values of the right $M F G \rightarrow$ left $M F G$, left calcarine $\rightarrow$ right calcarine, and positive $\mathrm{GC}$ values of the right $\mathrm{MFG} \rightarrow$ right calcarine and left calcarine $\rightarrow$ left $\mathrm{MFG}$, which was opposite to those in the HC group (Figure 5C). For old people, bidirectional connectivity between the left ITG and left MCC was significantly different to the $\mathrm{HC}$ 


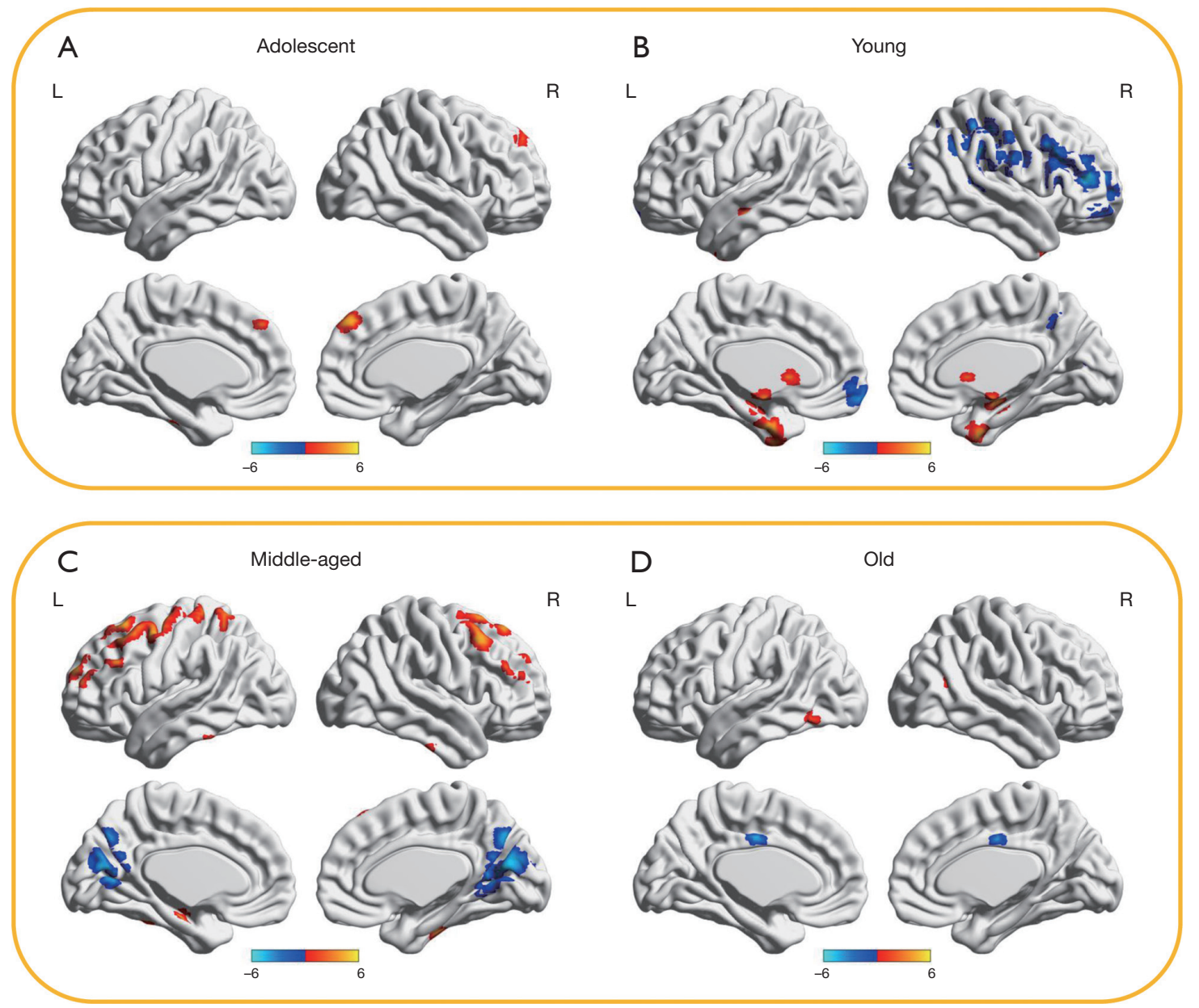

Figure 4 Brain regions showing different fALFF values between the MDD and HC groups at different age-onset (adolescents, young, middle-aged, old). (A) Regions showing significant inter-group differences in the adolescent group; (B) regions showing significant intergroup differences in the young group; (C) the increased and decreased fALFF for patients with middle-age onset relative to HC; (D) fALFF differences between MDD patients and HC in the old group. The warm color represents the higher fALFF of MDD compared to HC. The cold color represents the lower fALFF of MDD compared to HC. fALFF, fractional amplitude of low frequency fluctuations; MDD, major depressive disorder; HC, healthy controls.

group (Figure 5D).

\section{Discussion}

In this study, we uncovered the different fALFF trends over an entire lifespan in MDD and HC groups based on GLM. The mechanisms of different fALFF modes during the development, maturity, and aging process were explored using two-sample $t$-tests and subsequent GCA.

Concretely, the results obtained from the one-sample t-test showed that fALFF values in the left MSFG, right precuneus, PCC, MCC, left ACC, and SMA varied over the lifespan of patients in the MDD group, and was similar to those of the HC group. Furthermore, the regions detected both in the MDD and the HC groups were selected as ROIs. GLM was applied to model the fALFF with age or age $^{2}$ as predictors, along with sex. However, the fALFF showed no significant age effects in the MDD group. The fALFF values in the right precuneus, left PCC, MCC, left $\mathrm{AG}$, and right SMG followed positive quadratic trajectories over the age range. The two-sample $t$-test revealed discrepant inter-group effects at different age stages, which may account for the abnormal lifespan brain trajectories of the MDD group. Equally, GCA analysis indicated an 
Table 4 Regions with increased and decreased fALFF in patients with MDD relative to HC

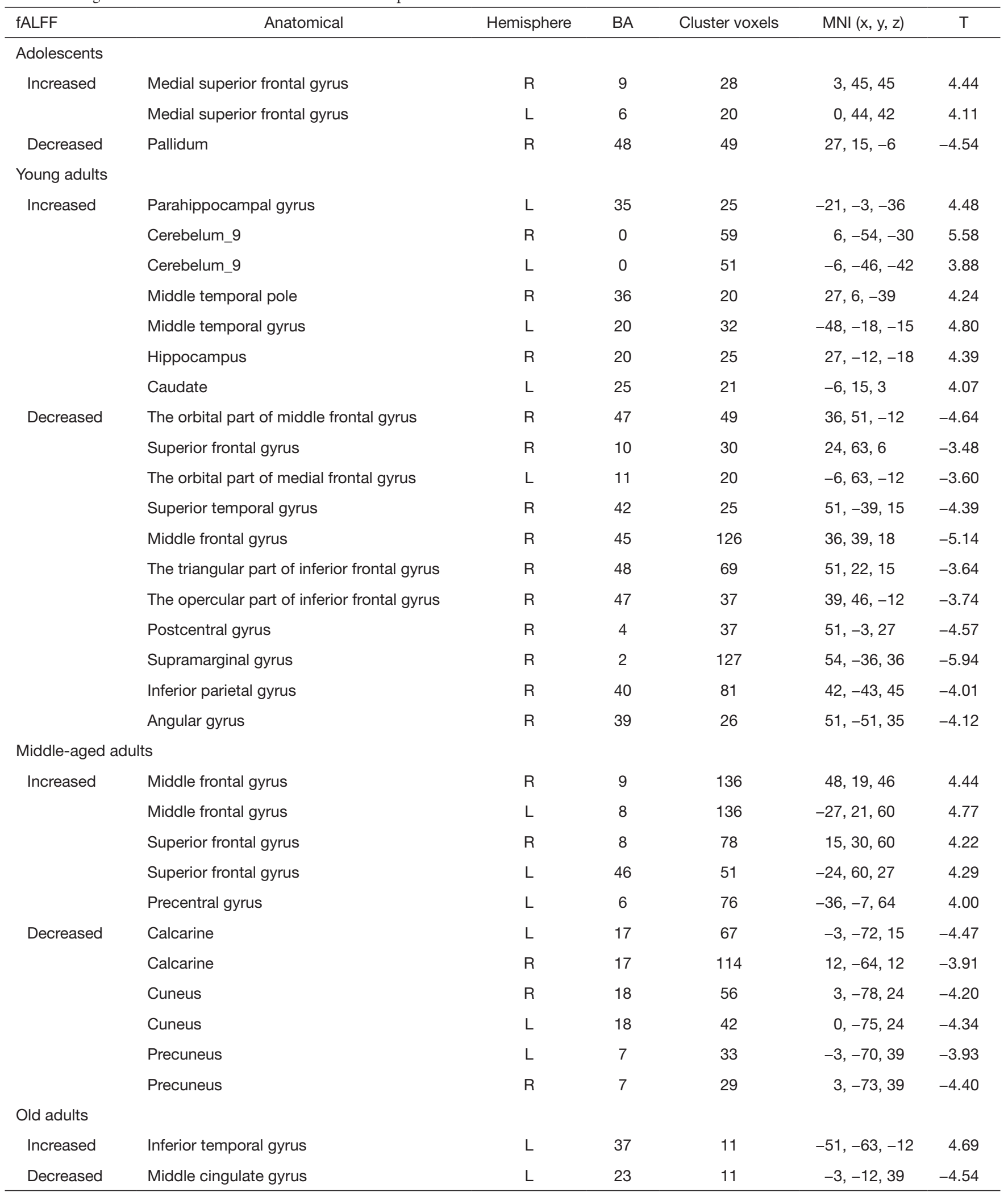

BA, Brodmann area; fALFF, fractional amplitude of low frequency fluctuations; MDD, major depressive disorder; HC, healthy controls. 
A

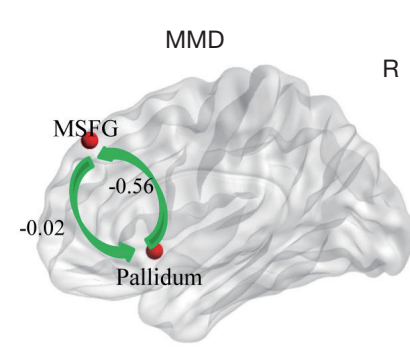

Adolescent

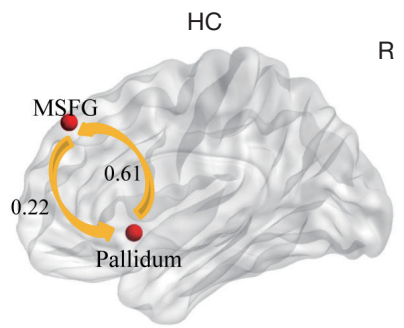

B

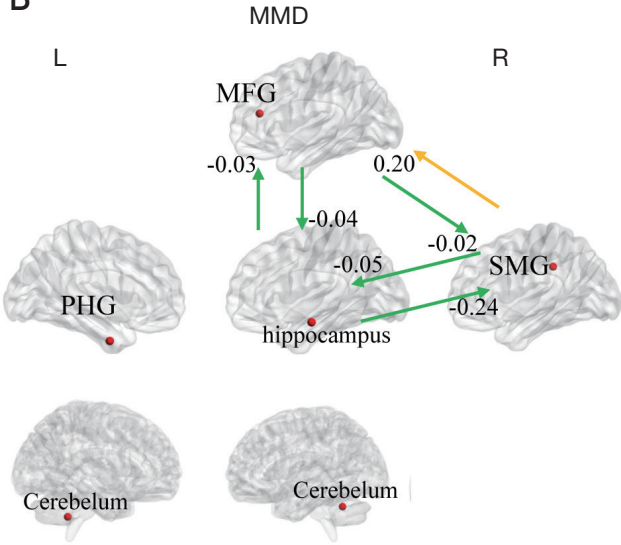

Young

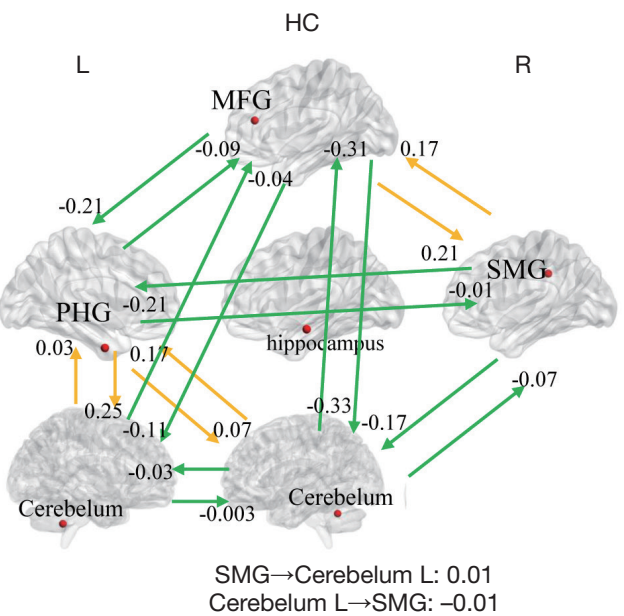

C

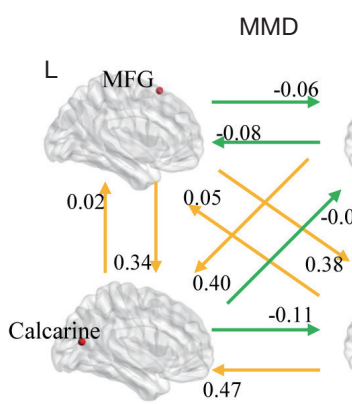

D

MMD

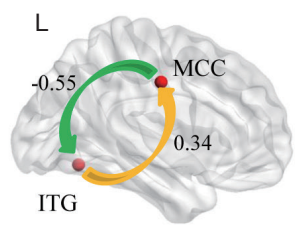

HC

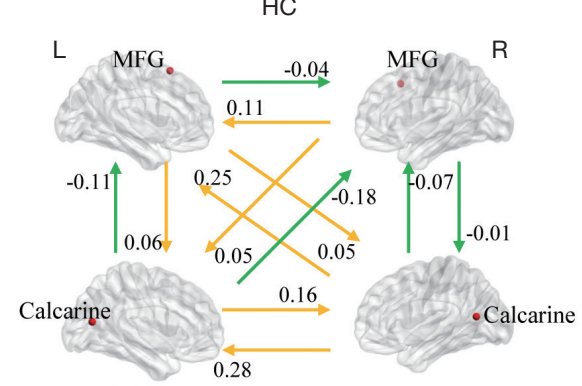

Old

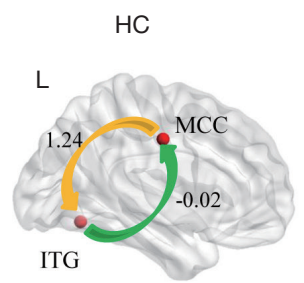

Figure 5 The inter-group comparison of GC flows with different ages of onset (adolescent, young adults, middle-aged adults, and old adults). (A) The differential GC flows for adolescents between the two groups, left: MDD, right: HC; (B) the inter-group comparison of GC flows for young people, left: MDD, right: HC, the GC flows between the right SMG and left cerebellum were not shown in the figure; (C) the differential GC flows for middle-aged people, left: MDD, right: HC; (D) differential GC flows in the old group, left: MDD, right: HC. Green/yellow arrows indicate significantly greater inhibition/activation of subsequent target activity. All GC flows are significantly different from zero ( $\mathrm{P}<0.05$, FDR-corrected). GC, Granger causality; MDD, major depressive disorder; HC, healthy controls. 
altered connectivity mode in MDD patients relative to HC at different ages of onset.

\section{Brain regions over a lifespan}

For HC, the four age groups (adolescent, young adults, middle-aged adults, and old adults) showed consistent fALFF changes in the right MSFG, precuneus, left cuneus, left PCC, left MCC, AG, right SMG, and left IPG (Figure 1B). These regions were mostly involved in a default mode network (DMN) whose activity increases in the absence of a task and is altered by development, aging, or cognitive impairment (26). The activation and graphical network metrics in these regions have been shown to be age-related over the lifespan of healthy people $(27,28)$. For MDD patients, the left MSFG, right precuneus, PCC, MCC, left ACC, and SMG exhibited consistent fALFF changes (Figure 1A). The brain regions were predominantly located in the DMN, which was the neural basis for the self-reference processing of depression. Improved activation or resting-state functional connectivity within the DMN were widely observed after improving depression symptoms by drug or cognitive therapy (29). In addition, the observed regional changes were similar to those in the HC group. This suggests that the correlation between fALFF and age may be detected in these regions over a patient's lifespan.

\section{Abnormal lifespan trajectory in MDD}

To examine the influence of age on fALFF, we computed GLM with age as a continuous variable in brain regions, which appeared both in the MDD and HC groups. For the HC group, a positive quadratic age-related effect in fALFF was evident in the right precuneus, left PCC, MCC, left AG, and right SMG. Over an adult lifespan, negative quadratic age effects (inverted- $U$ shaped) in the superior frontal cortex, IPG, and SMG, as well as a linear decreasing age effect in the precuneus, have been found using a weighted degree centrality metric (27). Age-related increases in the activation of the MCC were also uncovered (28). In this study, positive quadratic age effects were observed due to different network indicators. In contrast, there were no significant age effects of these regions in the MDD group, suggesting that MDD followed an abnormal development, maturational, and aging process. This finding coheres with previous reports on depression in different age stages (9). For adolescents with MDD, lower total surface area and regional reductions were observed in frontal regions, primary and higher order visual areas, and other ROIs. Adults with MDD had thinner cortical gray matter than HC in the orbitofrontal cortex, ACC, PCC, and other regions (19). Although depression is not necessarily irremediable, given that brain continues to develop and mature, the disrupted function plasticity occurred over the lifespan of the patient (30).

\section{Varied functional patterns with different age of onset}

The results of the two-sample $t$-test displayed differential inter-group effects at different ages of onset, which suggests that MDD may impact brain functions in a highly dynamic way.

For adolescents, inter-group effects were detectable in the MSFG and right pallidum, which is involved in prefrontal subcortical circuits (Figure 2A) (31). A previous study reported that adolescents with MDD (under 18 years old) have higher ALFF in the PCC, left ITG, right superior temporal gyrus, right insula, and other regions compared to $\mathrm{HC}(\mathrm{P}<0.005)$ (32). No group differences were observed in these regions in our study. This is probably because we applied stricter map thresholds and correction methods. Furthermore, the cut-off age and functional indicators were discrepant.

For young adults, the MDD group showed increased fALFF values in limbic regions (cerebellum, MTG, PHG and right hippocampus) compared with the HC group (Figure 2B). The cerebellum and PHG have been consistently implicated in typical emotion processing (33); the PHG is involved in establishing contextual information and the cerebellum plays an important in role in the perception of emotional stimuli (34). The hippocampus, as a hub of limbic circuits, suffers from stress (35) and plays an important role in emotional regulation, as well as memory formation and retrieval (2). Higher coherence-based regional homogeneity in the cerebellum has been found in MDD patients compared to HC (9). Hypoactivation was more widely detected in the PHG and hippocampus in MDD patients compared to HC (36,37). Evidence from a task-related study indicated that when MDD processed negative emotional stimuli relative to a neutral baseline, increased activation was observed in the cerebellum, PHG, MTG, and hippocampus. Conversely, when MDD processed positive emotional stimuli, decreased activity was seen in the cerebellum, PHG, and hippocampus (38). This demonstrates that MDD patients pay more attention to negative emotional stimuli relative to neutral stimuli 
and suffer from more severe emotional defects. Decreased fALFF values were mainly located in the right frontoparietal network (FPN), which consists of the dorsolateral frontal cortex (DLPFC), inferior frontal gyrus (IFG), and parietal regions. The dysfunction of FPN may result in changes in emotional reactivity and regulation (39). During matching negative faces relative to stimuli shape, decreased activation of DLPFC and IFG were found in young adults with MDD compared to HC (40). This indicated a coupling between the limbic regions and FPN.

For middle-aged people, increased fALFF values emerged in the MFG, SFG, and left precentral gyrus. The decreased fALFF emerged in the calcarine, cuneus, and precuneus in the MDD group as opposed to the HC group (Figure 2C). Compared with young patients, depression mainly affected the left-side frontal regions in middle-aged patients. The MFG (BA 9 and 46) is located in the DLPFC, and is recruited in cognitive functions such as memory and attention. The left MFG has an inhibitive and recalling function in psychological disease $(41,42)$. The MFG (BA 8) and precentral gyrus (BA6) were regarded to be frontal eye fields associated with eye movements in attention and memory (43). The increased activity in frontal regions may reflect the impaired attention and memory function for MDD patients.

The significantly reduced node degree and activity have been widely observed in the calcarine, cuneus, and precuneus (44). Additionally, the fALFF in the cuneus, occipital gyrus, and calcarine were decreased by depressive symptoms in patients with mild cognitive impairment (45). As a key hub of DMN, the precuneus was recruited in visual imagery, episodic memory, and self-directed operations, and has consistently been reported to be a neural basis of depression (44). The calcarine and cuneus are located in the occipital lobe and are related to the processing of visual information (46). Moreover, the calcarine sulcus is implicated in processing affective and sensory stimuli (47). Thus, the lower activation of the calcarine in MDD patients could be related to the lower tolerance threshold to the affective stimuli (48).

For patients with an elderly-onset, higher fALFF in the left ITG and lower fALFF in the left MCC were observed (Figure 2D). Dysfunction of the ITG has been linked to disrupted working memory (49), and higher ALFF in the ITG has been demonstrated by previous studies (50).

Significant regional differences were found in intergroup effects (MDD vs. HC) at different ages of onset. This suggested that MDD patients have widespread functional alterations compared to $\mathrm{HC}$, and that the patterns of alterations varied at different stages of life.

\section{Varied GC flows between MDD and HC}

For adolescents with MDD, the pattern of GC flows was similar to that of the HC group, except for the strength and direction (Figure 5). In contrast to HC, bidirectional prediction of negative activity was observed between the right MSFG and right pallidum. The MSFG and pallidum were recruited in prefrontal subcortical circuits (31), which are responsible for perception, the cognitive control of emotions, as well as internal and external attention. The disturbed GC flows of MDD might contribute to the cognitive and affective abnormalities in depression.

For young patients, the left PHG, left cerebellum, and right cerebellum have no significant GC flows with other ROIs. The cerebellum is functionally and structurally connected to the hippocampus and amygdala within the limbic system. This abnormal connectivity between the cerebellum and hippocampus has been identified in a previous study (51). Meanwhile, MDD displayed abnormal perception of emotional stimuli and establishment of contextual information (34), which indicated that the higher-order regions have lost control of the cerebellum and PHG. In addition, the right MFG predicted subsequent decreased activity in the right SMG for MDD patients, suggesting decreased top-down connectivity from the MFG to SMG and reduced shifting of attention for negative emotional stimuli (52).

For middle-aged patients, the right MFG (BA 9) predicted a decrease in activity in the left MFG (BA 8), and an increase in the right calcarine. This is in line with the mood-congruent biases in memory tasks, demonstrating the biases towards the retrieval of negative material, specifically during the recollection of negative personal memories or the false generation of these memories (10). Additionally, the left MFG (BA 8) is associated with saccadic eye movements (53); the predictive increased activity from the left calcarine to the left MFG may imply that more information was sent to the left MFG.

For patients with elderly-onset, the left ITG predicted increased activity in the left MCC, and the left MCC predicted decreased activity in the left ITG, which was opposite to those of the HC. Dysfunction of the ITG has been linked to disrupted working memory and vulnerability of the posterior-MCC has been shown in depression and post-traumatic stress disorder (52). Increased posterior- 
MCC activity has been observed when depression patients preformed a high memory load task(2-back) with neutral face distracters, suggesting abnormal recruitment of attention-control circuitry (54). Thus, the abnormal GC flows between the left ITG and the left MCC reflect the memory dysfunction in elderly people.

\section{Conclusions}

The present study provides an initial benchmark for tractspecific trajectories of functional plasticity abnormalities in MDD. Our findings demonstrated that widely distributed deficits of functional plasticity emerged over a lifespan relative to HC. This suggests that MDD has abnormal neurodevelopment, maturational, and aging processes compared to HC. The regional analysis revealed differential patterns of functional alterations with different ages of onset. For adolescents, inter-group effects occurred in prefrontal subcortical circuits. For young adults, inter-group effects occurred in limbic regions and the FP network. Furthermore, the differential granger information flows in MDD and $\mathrm{HC}$ indicated the underlying neural mechanism of regional diversity with different ages of onset. Our findings provide a new and comprehensive insight for the variability of neural mechanisms with ages of onset in MDD.

\section{Limitations}

Although this study explored the age-related relevance between the functional plasticity of the brain over a lifespan, there were two noteworthL Yangmitations to our investigation. Firstly, in the MDD group, the sex ratio was female-biased and the sample size of the elderly cohort was small. Secondly, some of the elderly subjects were receiving antidepressants. Given the medication treatment effects on $\mathrm{DMN}$, future studies with drug-naive patients are required to eliminate these effects.

\section{Acknowledgments}

Funding: The research was supported by the Chongqing Technology and Innovation Program of Social and Livelihood (cstc2016shmszx130014, HHZ).

\section{Footnote}

Reporting Checklist: The authors have completed the MDAR reporting checklist. Available at http://dx.doi.org/10.21037/ atm-21-367

Conflicts of Interest: All authors have completed the ICMJE uniform disclosure form (available at http://dx.doi. org/10.21037/atm-21-367). The authors have no conflicts of interest to declare.

Ethical Statement: The authors are accountable for all aspects of the work in ensuring that questions related to the accuracy or integrity of any part of the work are appropriately investigated and resolved. Written informed consent was obtained from all participants at their local institution. The analysis in this study was approved by the REST-meta-MDD consortium (approval ID: U0040). The study was conducted in accordance with the Declaration of Helsinki (as revised in 2013).

Open Access Statement: This is an Open Access article distributed in accordance with the Creative Commons Attribution-NonCommercial-NoDerivs 4.0 International License (CC BY-NC-ND 4.0), which permits the noncommercial replication and distribution of the article with the strict proviso that no changes or edits are made and the original work is properly cited (including links to both the formal publication through the relevant DOI and the license). See: https://creativecommons.org/licenses/by-nc-nd/4.0/.

\section{References}

1. Yao Z, Wang L, Lu Q, et al. Regional homogeneity in depression and its relationship with separate depressive symptom clusters: a resting-state fMRI study. J Affect Disord 2009;115:430-8.

2. Price RB, Duman R. Neuroplasticity in cognitive and psychological mechanisms of depression: an integrative model. Mol Psychiatry 2020;25:530-43.

3. Zhou HX, Chen X, Shen YQ, et al. Rumination and the default mode network: Meta-analysis of brain imaging studies and implications for depression. Neuroimage 2020;206:116287.

4. Wang L, Li K, Zhang QE, et al. Interhemispheric functional connectivity and its relationships with clinical characteristics in major depressive disorder: a resting state fMRI study. PLoS One 2013;8:e60191.

5. Kaiser RH, Andrews-Hanna JR, Wager TD, et al. Largescale network dysfunction in major depressive disorder: a meta-analysis of resting-state functional connectivity. JAMA Psychiatry 2015;72:603-11. 
6. Tsang A, Lebel CA, Bray SL, et al. White matter structural connectivity is not correlated to cortical resting-state functional connectivity over the healthy adult lifespan. Front Aging Neurosci. 2017;9:144.

7. Coupé P, Manjón JV, Lanuza E, et al. Lifespan changes of the human brain in Alzheimer's disease. Sci Rep 2019;9:3998.

8. Baselmans BM, Willems YE, van Beijsterveldt C, et al. Unraveling the genetic and environmental relationship between well-being and depressive symptoms throughout the lifespan. Front Psychiatry 2018;9:261.

9. Liu F, Hu M, Wang S, et al. Abnormal regional spontaneous neural activity in first-episode, treatmentnaive patients with late-life depression: a resting-state fMRI study. Prog Neuropsychopharmacol Biol Psychiatry 2012;39:326-31.

10. Holt RJ, Graham JM, Whitaker KJ, et al. Functional MRI of emotional memory in adolescent depression. Dev Cogn Neurosci 2016;19:31-41.

11. Bellaera L, von Mühlenen A. The effect of induced sadness and moderate depression on attention networks. Cogn Emot 2017;31:1140-52.

12. Biswal BB. Resting state $\mathrm{fMRI}$ : a personal history. Neuroimage 2012;62:938-44.

13. Zang YF, He Y, Zhu CZ, et al. Altered baseline brain activity in children with ADHD revealed by resting-state functional MRI. Brain Dev 2007;29:83-91.

14. Bu X, Hu X, Zhang L, et al. Investigating the predictive value of different resting-state functional MRI parameters in obsessive-compulsive disorder. Transl Psychiatry 2019;9:17.

15. Zou QH, Zhu CZ, Yang Y, et al. An improved approach to detection of amplitude of low-frequency fluctuation (ALFF) for resting-state fMRI: fractional ALFF. J Neurosci Methods 2008;172:137-41.

16. Vij SG, Nomi JS, Dajani DR, et al. Age-related changes in spatial and temporal features of resting state fMRI. bioRxiv 2017:109181.

17. Yan CG, Chen X, Li L, et al. Reduced default mode network functional connectivity in patients with recurrent major depressive disorder. Proc Natl Acad Sci U S A 2019;116:9078-83.

18. Kang L, Zhang A, Sun N, et al. Functional connectivity between the thalamus and the primary somatosensory cortex in major depressive disorder: a resting-state fMRI study. BMC Psychiatry 2018;18:339.

19. Schmaal L, Hibar D, Sämann P, et al. Cortical abnormalities in adults and adolescents with major depression based on brain scans from 20 cohorts worldwide in the ENIGMA Major Depressive Disorder Working Group. Mol Psychiatry 2017;22:900-9.

20. Hedden T, Gabrieli JD. Insights into the ageing mind: a view from cognitive neuroscience. Nat Rev Neurosci 2004;5:87-96.

21. Hamilton JP, Chen G, Thomason ME, et al. Investigating neural primacy in Major Depressive Disorder: multivariate Granger causality analysis of resting-state fMRI timeseries data. Mol Psychiatry 2011;16:763-72.

22. Yan C-G, Craddock RC, Zuo XN, et al. Standardizing the intrinsic brain: towards robust measurement of interindividual variation in 1000 functional connectomes. Neuroimage 2013;80:246-62.

23. Lee SW, Kim E, Chung Y, et al. Believing is seeing: an fMRI study of thought-action fusion in healthy male adults. Brain Imaging Behav 2021;15:300-10.

24. Jiang K, Wang J, Zheng A, et al. Amplitude of lowfrequency fluctuation of resting-state $\mathrm{fMRI}$ in primary nocturnal enuresis and attention deficit hyperactivity disorder. Int J Dev Neurosci 2020;80:235-45.

25. Jiang $Y$, Tian $Y$, Wang Z. Causal interactions in human amygdala cortical networks across the lifespan. Sci Rep 2019;9:5927.

26. Dennis EL, Thompson PM. Functional brain connectivity using fMRI in aging and Alzheimer's disease. Neuropsychol Rev 2014;24:49-62.

27. Cao M, Wang JH, Dai ZJ, et al. Topological organization of the human brain functional connectome across the lifespan. Dev Cogn Neurosci 2014;7:76-93.

28. Kennedy KM, Rodrigue KM, Bischof GN, et al. Age trajectories of functional activation under conditions of low and high processing demands: an adult lifespan fMRI study of the aging brain. Neuroimage 2015;104:21-34.

29. Carhart-Harris RL, Roseman L, Bolstridge M, et al. Psilocybin for treatment-resistant depression: fMRImeasured brain mechanisms. Sci Rep 2017;7:13187.

30. Stickel S, Wagels L, Wudarczyk O, et al. Neural correlates of depression in women across the reproductive lifespanAn fMRI review. J Affect Disord 2019;246:556-70.

31. Vataja R, Pohjasvaara T, Leppävuori A, et al. Magnetic resonance imaging correlates of depression after ischemic stroke. Arch Gen Psychiatry 2001;58:925-31.

32. Gong Y, Hao L, Zhang X, et al. Case-control resting-state fMRI study of brain functioning among adolescents with first-episode major depressive disorder. Shanghai Arch Psychiatry 2014;26:207.

33. Lindquist KA, Wager TD, Kober H, et al. The brain 
basis of emotion: a meta-analytic review. Behav Brain Sci 2012;35:121.

34. Schmahmann JD. The role of the cerebellum in affect and psychosis. J Neurolinguistics 2000;13:189-214.

35. McEwen BS, Nasca C, Gray JD. Stress effects on neuronal structure: hippocampus, amygdala, and prefrontal cortex. Neuropsychopharmacology 2016;41:3-23.

36. Bürger C, Redlich R, Grotegerd D, et al. Differential abnormal pattern of anterior cingulate gyrus activation in unipolar and bipolar depression: an fMRI and pattern classification approach. Neuropsychopharmacology 2017;42:1399-408.

37. Chen CH, Suckling J, Lennox BR, et al. A quantitative meta-analysis of fMRI studies in bipolar disorder. Bipolar Disord 2011;13:1-15.

38. Groenewold NA, Opmeer EM, de Jonge P, et al. Emotional valence modulates brain functional abnormalities in depression: evidence from a meta-analysis of fMRI studies. Neurosci Biobehav Rev 2013;37:152-63.

39. Salas CE, Radovic D, Yuen KS, et al. "Opening an emotional dimension in me": Changes in emotional reactivity and emotion regulation in a case of executive impairment after left fronto-parietal damage. Bull Menninger Clin 2014;78:301-34.

40. Li H, Wei D, Sun J, et al. Fronto-limbic alterations in negatively biased attention in young adults with subthreshold depression. Front Psychol 2017;8:1354.

41. Fitzgerald PB, Srithiran A, Benitez J, et al. An fMRI study of prefrontal brain activation during multiple tasks in patients with major depressive disorder. Human Brain Mapping 2008;29:490-501.

42. Xu Z, Zhang J, Wang D, et al. Altered brain function in drug-naïve major depressive disorder patients with early-life maltreatment: a resting-state fMRI study. Front Psychiatry 2019;10:255.

43. Hung J, Driver J, Walsh V. Visual selection and the human frontal eye fields: effects of frontal transcranial magnetic stimulation on partial report analyzed by Bundesen's theory of visual attention. J Neurosci 2011;31:15904-13.

44. Li G, Rossbach K, Zhang A, et al. Resting-state functional changes in the precuneus within first-episode drugnaive patients with MDD. Neuropsychiatric disease and treatment 2018;14:1991.

45. Yu Y, Li Z, Lin Y, et al. Depression affects intrinsic brain activity in patients with mild cognitive impairment. Front
Neurosci 2019;13:1333.

46. Cai L, Liang Y, Huang H, et al. Cerebral functional activity and connectivity changes in anti-N-methyl-Daspartate receptor encephalitis: A resting-state fMRI study. Neuroimage Clin 2020;25:102189.

47. Wei HL, Zhou X, Chen YC, et al. Impaired intrinsic functional connectivity between the thalamus and visual cortex in migraine without aura. J Headache Pain 2019;20:116.

48. Zhu Y, Song X, Xu M, et al. Impaired interhemispheric synchrony in Parkinson's disease with depression. Sci Rep 2016;6:27477.

49. Zeng LL, Shen H, Liu L, et al. Identifying major depression using whole-brain functional connectivity: a multivariate pattern analysis. Brain 2012;135:1498-507.

50. Guo WB, Liu F, Xue ZM, et al. Alterations of the amplitude of low-frequency fluctuations in treatmentresistant and treatment-response depression: a resting-state fMRI study. Prog Neuropsychopharmacol Biol Psychiatry 2012;37:153-60.

51. Gudayol-Ferré E, Peró-Cebollero M, González-Garrido AA, et al. Changes in brain connectivity related to the treatment of depression measured through fMRI: a systematic review. Front Hum Neurosci 2015;9:582.

52. Hilland E, Landrø NI, Harmer CJ, et al. Attentional bias modification is associated with $\mathrm{fMRI}$ response toward negative stimuli in individuals with residual depression: a randomized controlled trial. J Psychiatry Neurosci 2020;45:23.

53. Vernet M, Quentin R, Chanes L, et al. Frontal eye field, where art thou? Anatomy, function, and non-invasive manipulation of frontal regions involved in eye movements and associated cognitive operations. Front Integr Neurosci 2014;8:66.

54. Vogt BA. Midcingulate cortex: structure, connections, homologies, functions and diseases. J Chem Neuroanat 2016;74:28-46.

(English Language Editor: A. Kassem)

Cite this article as: Yang L, Wei AH, Ouyang TT, Cao ZZ, Duan AW, Zhang HH. Functional plasticity abnormalities over the lifespan of first-episode patients with major depressive disorder: a resting state fMRI study. Ann Transl Med 2021;9(4):349. doi: 10.21037/atm-21-367 